\title{
Nature of Dislocations in Silicon
}

Hansen, Lars Bruno; Stokbro, Kurt; Lundqvist, Bengt; Jacobsen, Karsten Wedel; Deaven, D. M.

\section{Published in:}

Physical Review Letters

Link to article, DOI:

10.1103/PhysRevLett.75.4444

Publication date:

1995

Document Version

Publisher's PDF, also known as Version of record

Link back to DTU Orbit

Citation (APA):

Hansen, L. B., Stokbro, K., Lundqvist, B., Jacobsen, K. W., \& Deaven, D. M. (1995). Nature of Dislocations in Silicon. Physical Review Letters, 75(24), 4444-4447. https://doi.org/10.1103/PhysRevLett.75.4444

\section{General rights}

Copyright and moral rights for the publications made accessible in the public portal are retained by the authors and/or other copyright owners and it is a condition of accessing publications that users recognise and abide by the legal requirements associated with these rights.

- Users may download and print one copy of any publication from the public portal for the purpose of private study or research.

- You may not further distribute the material or use it for any profit-making activity or commercial gain

- You may freely distribute the URL identifying the publication in the public portal

If you believe that this document breaches copyright please contact us providing details, and we will remove access to the work immediately and investigate your claim 


\title{
Nature of Dislocations in Silicon
}

\author{
L. B. Hansen, ${ }^{1}$ K. Stokbro, ${ }^{2}$ B. I. Lundqvist, ${ }^{1}$ K. W. Jacobsen, ${ }^{3}$ and D. M. Deaven ${ }^{4}$ \\ ${ }^{1}$ Department of Applied Physics, Chalmers University of Technology and Goteborg University, S-41296 Goteborg, Sweden \\ ${ }^{2}$ Scuola Internazionale Superiore di Studi Avanzati, Via Beirut 4, I-34014 Trieste, Italy \\ ${ }^{3}$ Center for Atomic-scale Materials Physics and Physics Department, Technical University of Denmark, DK-2800 Lyngby, Denmark \\ ${ }^{4}$ Ames Laboratory, Ames, Iowa 50011
}

(Received 16 June 1995)

\begin{abstract}
Interaction between two partial $90^{\circ}$ edge dislocations is studied with atomic-scale simulations using the effective-medium tight-binding method. A large separation between the two dislocations (up to $30 \AA$ ), comparable to experimental values, is achieved with a solution of the tight-binding Hamiltonian that scales linearly with the number of atoms. The partial edge dislocation is found to be very accurately described by the Peierls-Nabarro dislocation model, with generalized stacking-fault restoring forces, as reflected both in the interaction energy and in the displacement field. An asymmetric core reconstruction provides fourfold coordination, making Si behave elastically down to atomic distances.
\end{abstract}

PACS numbers: 61.72.Lk, 71.10.+x

Dislocations are of key importance for mechanical properties of materials, e.g., their plasticity. For the description of dislocations the Peierls-Nabarro (PN) model $[1,2]$ plays a leading role. This model accounts for the dual nature of a dislocation by using continuum elasticity theory to describe two displaced semi-infinite crystals and including the effect of the discrete crystal lattice by considering a restoring force for the interface between the two crystals. The PN model does not only give analytical expressions for the dislocation energy outside the core region of the dislocation. It also makes assumptions about the energy in the core region. This makes the PN model very useful for practical applications; recent works include calculations of the critical thickness of strained layers and the Peierls stress [3]. In these applications the emphasis is on the need to include the effects of the dislocation core and to remove the artificial core cutoff radius present in elasticity theory. This is accomplished in the PN model. Outstanding questions concern its atomic-scale basis and the relative extents of the core and elastic regions of a dislocation. While the dominance of elastic forces has always been evident for the long-range behavior, the short-range properties have been an open question.

Experimental observations [4] imply that dislocations in $\mathrm{Si}$ belonging to the glide sets are dissociated into partial dislocations bounding stacking faults with sizes ranging from about 30 to $50 \AA$. The understanding of the nature of such extended defects and of their interactions on an atomic level is hampered by the large number $N$ of atoms involved. Systems with similar complexity have been treated with standard first-principles methods [5]; however, such calculations are computationally too intensive to be suitable as a general approach, because these grow cubically, $O\left(N^{3}\right)$, with the size of the system.

This Letter reports results of a many-atom calculation of ground-state configurations and energies for the $90^{\circ}$ partial edge dislocation in $\mathrm{Si}$. The effective-medium tight-binding (EMTB) model [6] and the $O(N)$ method by Goedecker and Colombo [7] are used to calculate the energetics. The result is compared with that of the PN model using general stacking-fault (GSF) restoring forces.

Recent atomic-scale descriptions of dislocations in $\mathrm{Si}$ have concerned (i) the GSF surface for the two important glide planes in Si [8], (ii) its application to the PN model [9], (iii) the interactions of dislocations in Si belonging to the shuffle set [5] for length scales, where elastic interactions become valid, and (iv) the core structure for the $90^{\circ}$ edge dislocation, found to be reconstructed [10].

This study is novel by giving a full atomic-scale support for both the elastic and core parts of the PN model, with a realistic quantum-mechanical potential. Preliminary results for the mobiliy of the partial edge dislocation are given in Ref. [11].

The dislocation structure of $\mathrm{Si}$ has been discussed in many excellent reviews (see, e.g., Refs. [12] and [13]). Because the diamond cubic structure is formed by two face-centered-cubic (fcc) lattices, dislocations are expected to be similar to the fcc ones. In Si the main slip plane is the (111) plane and the major slip direction is [110].

The diamond lattice allows for two distinct locations of the slip plane, placed between atomic planes that are separated by either (a) a nearest-neighbor $(n-n)$ distance (planes with the same index), the so-called "shuffle" slip plane, or (b) one-third of a $n-n$ distance (planes of different indices), the "glide" slip plane. The perfect glide dislocation is known to dissociate into partial dislocations, following the reaction $\frac{1}{2}\langle\overline{1} 0 \overline{1}\rangle \rightarrow \frac{1}{6}\langle\overline{2} 11\rangle+\frac{1}{6}\langle\overline{1} \overline{1} 2\rangle$. For the dislocation line along the $\langle 1 \overline{1} 0\rangle$ direction, the latter glide $\left(\frac{1}{6}\langle\overline{1} \overline{1} 2\rangle\right)$ forms the $90^{\circ}$ edge dislocation (Fig. 1).

For the core structure of the edge dislocation several models have been proposed: (i) a symmetric reconstruction [14], where the atoms with the dangling bonds move closer together and form "quasi-fivefold" coordinated atoms [Fig. 1(a)], and (ii) an asymmetric one $[15,16]$, where the symmetry along the dislocation line is 
(a)

(b)

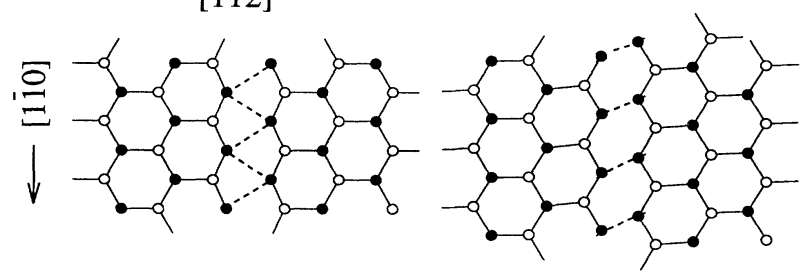

FIG. 1. Atomic structure projected on the (111) slip plane. Atoms immediately above (filled circles) and below the slip plane (open circles) are shown. In (a) the symmetric reconstruction is shown and in (b) the asymmetric reconstruction.

broken and the atoms in the core have retained their fourfold coordination [Fig. 1(b)]. This latter reconstruction has been found to be energetically more favorable than the symmetric one [10]. The EMTB model exhibits the same reconstruction, the asymmetric reconstruction being more stable by $0.18 \mathrm{eV} / \AA$, in very good agreement with the ab initio result by Bigger et al. [10] of $0.23 \mathrm{eV} / \AA$.

To reveal the nature of a dislocation one needs to know, in addition to its core structure, its effect at varying distances from the core. For this purpose we study the interaction of a dislocation antidislocation pair (dipole), a distance $R$ apart. Figure 2 shows the (111) glide plane for the two edge dislocations ( $R$ is here $27 \AA$ ), with opposite Burgers vectors. In our simulation we use a unit cell, which in the [1112] $(x)$ direction (parallel to the Burgers vector) has a length of $60 \AA$ in the [111] ( $y$ ) direction (normal to both the Burgers vector and the dislocation line) $40 \AA$, and in the [110] direction (along the dislocation line $)$ a length of $a / \sqrt{2}(a=5.41 \AA$, $a$ being the lattice constant) is used. The total number of atoms in the unit cell is 432 . We impose symmetry along the dislocation line and use a unit cell that only allows for

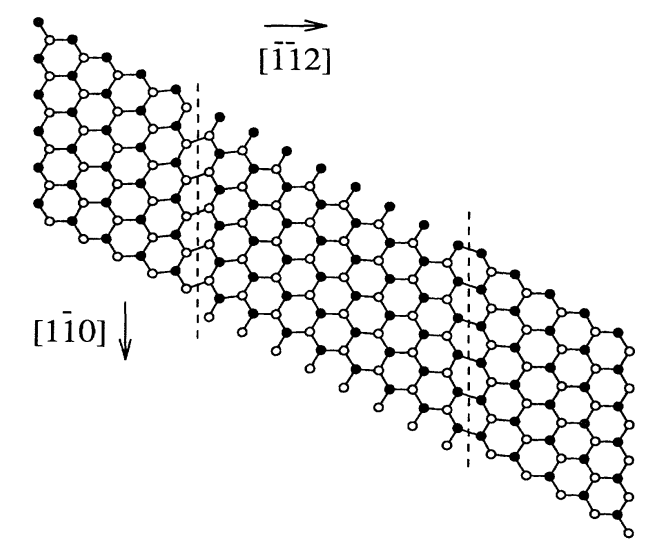

FIG. 2. The unit cell for the atomistic simulations in the (111) slip plane. The dislocation lines (dashed lines) are shown for each dislocation. The unit cell has been repeated six times in the direction of the dislocation lines. the reconstruction. Our real-space method uses a larger unit cell in this direction, avoiding problems with the $k$ point summation, but using the symmetry to reduce the computational effort.

The dislocations are introduced by imposing the isotropic elastic fields [2] from the dislocation array. The ground-state configuration is subsequently found using molecular-dynamics simulations at different temperatures, using a simulated-annealing technique, with $1000 \mathrm{~K}$ being the highest temperature.

In the simulations we use periodic-boundary conditions in all directions. Interaction between the dipoles in different repeated cells is a consequence of these boundary conditions. Because of the large unit cell this dipole-dipole interaction can be calculated using continuum elasticity theory [5]. If the stacking of the dislocation results in a tilt boundary, a grain boundary can be created along the boundary of the unit cell. To avoid this, we use the quadrupolar array, introduced by Bigger et al. [10]. The quadrupolar array also effectively removes problems with a tilt boundary, when the distance $R$ between the dislocations in the unit cell is decreased [17].

The EMTB total-energy method is based on the density-functional theory and is derived $a b$ initio, that is, without any fitting to experiment. The method is based on the effective-medium concept of a reference system [18]. The two main approximations are the use of a transferable charge density [19] and a first order linearized muffin-tin orbital (LMTO) [20] basis set. The parametrized LMTO-TB model gives the band structure in good agreement with the local-density approximation (LDA) result [21]. The EMTB model has been found to describe correctly bulk and surface properties for $\mathrm{Si}$, including the dimerization of the $\mathrm{Si}(100)$ surface [21].

The LMTO-TB Halmiltonian is solved using the $O(N)$ method by Goedecker and Colombo [7,22]. This method is particularly suited for systems containing band gap, such as insulators and semiconductors, because it is based on an approximation for the Fermi function. In terms of the tight-binding orbitals, $\phi_{l \alpha}$, centered on the $\alpha$ th atom, the $O(N)$ method band-structure energy $\left(E_{b s}\right)$ expression

$$
E_{b s}=\sum_{l \alpha, l^{\prime} \alpha^{\prime}}\left\langle\phi_{l \alpha}|H| \phi_{l^{\prime} \alpha^{\prime}}\right\rangle\left\langle\phi_{l^{\prime} \alpha^{\prime}}\left|f\left(\frac{H-\mu}{k T}\right)\right| \phi_{l \alpha}\right\rangle
$$

is evaluated efficiently by approximating the Fermi function $f$ by a polynomial expression in the range of the eigenvalues $\epsilon_{i}$ of the Hamiltonian $H$. This corresponds to inserting terms of the form $H^{n}$ into Eq. (1). The bandstructure energy can then be found by using projection operators. Exact forces can be found from the derivative of the approximate total energy, i.e., without relying on the Hellman-Feynman theorem, and thereby obtain exact energy conservation in a molecular-dynamics simulation.

For $\mathrm{Si}$ the EMTB band gap is $2.1 \mathrm{eV}$, which makes the physical properties less affected by a high electronic temperature in the Fermi function, which we have chosen 
to be $0.7 \mathrm{eV}$. With this temperature and a localization volume [7] for the projection operators with the radius $18 \mathrm{bohr}$, we obtain an accuracy of $5 \mathrm{meV} /$ atom compared to a standard diagonalization. In our implementation we use a constant number of electrons [17], instead of a constant chemical potential, as proposed in Ref. [7].

In agreement with Bigger et al. [10] we find that the symmetric reconstruction introduces states in the band gap [17], which are not present for the asymmetric reconstruction. This provides an explanation of the stability of the fourfold reconstruction. The tendency for fourfold coordination for the atoms in the dislocation core is important for the elastic behavior of the dislocation.

In the PN model the nonelastic displacements in the core region are assumed to be confined to the glide plane, separating two semi-infinite half planes. At each point in the glide plane the displacement $\mathbf{f}$ of the upper half $(y>0)$ with respect to the lower half plane $(y<0)$ is balanced by the restoring force $F(\mathbf{f})$.

Specific information for the crystal restoring forces can be introduced into the PN model by the use of the GSF model [23]. Here the energy for displacing the upper half plane with respect to the lower one is calculated. The energy for this, the so-called $\gamma(\mathbf{f})$ surface, gives $F(\mathbf{f})=$ $-\partial \gamma(\mathbf{f}) / \partial \mathbf{f}$. The $\gamma$ surface for the relevant displacement $\mathbf{f}$ in the [112] direction has been calculated according to the procedure used in Refs. [8,9]. The results for the maximum of the $\gamma(\mathbf{f})$ surface, the unstable stacking fault energy, $\gamma_{\max }$, and for the intrinsic stacking fault energy (after a displacement of the Burgers vector), $\gamma_{s f}$, are given in Table I. The EMTB and the $a b$ initio LDA methods are seen to give comparable results.

Assuming a sinusoidal relation between the restoring force $F$ and the displacement $\mathbf{f}$ one obtains a measure, $\xi$, of the region where the disregistry is larger than half the maximum, given by $\mu b^{2} /\left[(1-\nu) 4 \pi^{2} \gamma_{\max }\right]$ [17]. For the $\gamma(\mathbf{f})$ surface of $\mathrm{Si}$ the sinusoidal relation gives values for $\xi$ in close agreement with those obtained by Joós, Ren, and Duesbery [9], in their full solution of the PN model.

TABLE I. Parameters describing the dislocation in the Peierls-Nabarro model. The numbers in parentheses are from the $a b$ initio LDA values in Ref. [9].

\begin{tabular}{llc}
\hline \hline & \multicolumn{1}{c}{ EMTB } & Experimental $^{\mathrm{a}}$ \\
\hline Burgers vector $b(\AA)$ & 2.18 & 2.22 \\
$c_{11}$ (Mbar) & 1.43 & 1.66 \\
$c_{12}$ (Mbar) & 0.90 & 0.64 \\
$c_{44}$ (Mbar) & 0.90 & 0.80 \\
Poison ratio $\nu$ & 0.22 & 0.256 \\
Shear modulus $\mu\left(\mathrm{eV} / \AA^{3}\right)$ & 0.45 & 0.42 \\
Width of dislocation $\xi(\AA)$ & $0.44(0.58)$ & \\
Misfit energy $W_{m}$ & $0.25(0.21)$ & \\
$\gamma_{\text {max }}\left(\mathrm{eV} / \AA^{2}\right)$ & $0.16(0.12)$ & 0.006 \\
$\gamma_{\text {sf }}\left(\mathrm{eV} / \AA^{2}\right)$ & $0.005(.0 .006)$ & \\
\hline \hline
\end{tabular}

${ }^{\mathrm{a}}$ From Ref. [2].
The PN expression for the interaction energy can now be expressed in terms of $\xi[2]$,

$$
E_{\text {dipole }}^{\mathrm{PN}}(R)=W_{m}+\frac{\mu b^{2}}{2 \pi(1-\nu)} \ln \frac{R}{2 \xi}+\gamma_{\mathrm{sf}} R .
$$

The misfit energy in the glide plane, $W_{m}$, is given by $\mu b^{2} /[4 \pi(1-\nu)]$. Table I summarizes the parameters used in Eq. (2), with effective elastic constants $\mu$ and $\nu$ from a Voigt average [2] over the elastic constants for the EMTB potential. $b$ is the Burgers vector.

The total energy of the unit cell for the relaxed dipole is a sum of the energy of the isolated dipole [Eq. (2)] and of the dipole-dipole interaction energy [5]. The core energy does not enter the latter, because the net Burgers vector is zero for each dipole. Figure 3 shows the total energy (circles) for the unit cell, corrected for the dipole-dipole interaction, as a function of the separation $R$ between the two dislocations in the dipole. The PN result (solid line) from Eq. (2), with the EMTB parameters of Table I, is also shown. The atomic-scale description is seen to be very close to the the PN result. This close agreement is shown more directly in the displacement field around the dislocations. Figure 4(a) shows the atomic configuration around one partial dislocation in the unit cell. The displacements of the atoms in the Burgers vector direction $u_{x}(x, y)$, which is the most important one for the edge dislocation, relative to their positions in the perfect diamond lattice are shown in Fig. 4(b). The results from the atomic-scale simulation (circles) and from the PN model [2] (solid lines) are shown.

The PN model and the atomic simulation both predict a very narrow dislocation for $\mathrm{Si}$. The reason is the rigidity towards displacement, accounted for by a large value of $\gamma_{\max }$. In metallic systems the situation is different. A molecular-dynamics study for $\mathrm{Cu}$ [24] shows that the core is extended into the stacking-fault region, even at low temperature.

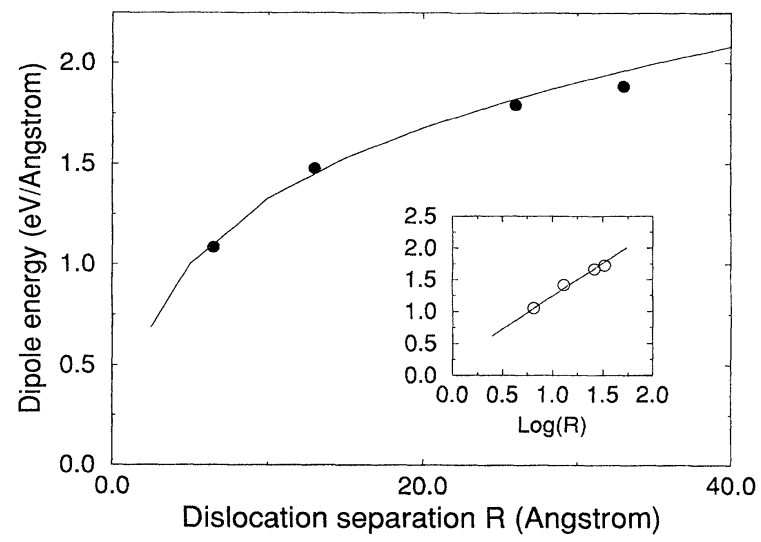

FIG. 3. The dislocation dipole energy from the atomic simulations (circles). The solid line is the result of the PN model, with parameters given in Table I. The inset shows the linear scaling with the logarithm of the distance between the dislocations. Here the linear contribution from the stacking fault energy has been removed. 


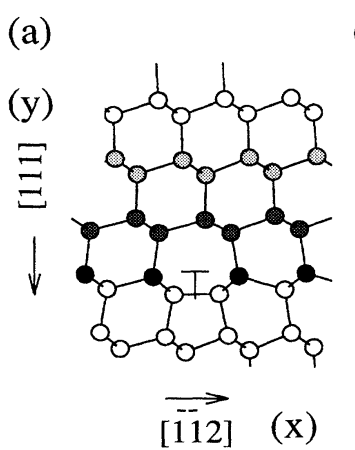

(b)

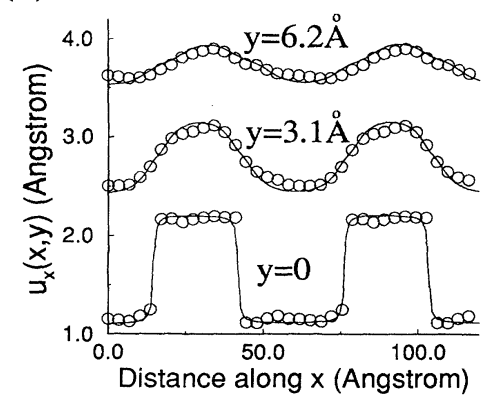

FIG. 4. (a) Atomic configuration around one partial dislocation. For the black atoms in the glide plane $(y=0)$, grey atoms $(y=3.1 \AA)$, and light-grey atoms $(y=6.2 \AA)$ the displacement field $u_{x}(x, y)$ is shown in (b). The circles are from the atomic simulation for $R=27 \AA$ (each circle represents an atom). The solid lines are the displacement field from the PN model. The periodic boundary conditions are used in the $x$ direction. The curves are displaced arbitrarily relative to each other.

For $\mathrm{Si}$ we find an elastic behavior close to the dislocation, i.e., $\mathrm{Si}$ approaches the continuum limit very fast, which confirms the conclusions by Arias and Joannopoulos [5] based on energy considerations (Fig. 3). This elastic behavior is seen directly in the displacement field in Fig. 4, which follows closely the results predicted by the continuum theory already in the atomic layer adjacent to the dislocation. The stress field around an edge dislocation in $\mathrm{Si}$ has been studied experimentally [25], showing the same elastic behavior, however, on a longer length scale.

The extra dipole energy for a quasi-fivefold coordination is $0.4 \mathrm{eV} / \AA$, assuming the same elastic interaction energy. This would result in a misfit energy significantly higher than the PN result. As discussed by Joós, Ren, and Duesbery [9], the PN misfit energy $W_{m}$ depend only on elastic properties. The restoring $\gamma$ surface determines the spreading of the energy in the glide plane. A configuration with no dangling bond is thus most likely to be described correctly in the PN model. Together with the elasticity of $\mathrm{Si}$, this gives a qualitative explanation of the agreement between the two methods for Si.

In summary, the glide edge dislocation studied by atomic-scale simulations is found to be described accurately by the PN model, using the GSF model for the interatomic interactions. The EMTB potential gives values for the PN dislocation width $\xi$ and misfit energy $W_{m}$ in good agreement with those of $a b$ initio calculations $[8,9]$. The assumptions of the PN model are found justified for $\mathrm{Si}$ because of its very elastic behavior. This nanoscale elasticity of Si (Fig. 4) is the key physical result of this study and is a direct result of the fact that the asymmetric reconstruction provides fourfold coordination for the atoms in the core.

Lars B. Hansen gratefully acknowledges support from The Swedish National Board for Ind. and
Tech. Development, The Swedish Natural Science Research Council and the EEC Contract No. CHRXCT93-0134. K.S. acknowledges the EEC Contracts No. ERBCHBGCT 920180 and No. ERBCHRXCT 930342 and the CNR project Supaltemp.

[1] R. E. Peierls, Proc. Phys. Soc. London 52, 34 (1940); F. R. N. Nabarro, Proc. Phys. Soc. London 59, 256 (1947).

[2] J.P. Hirth and J. Lothe, Theory of Dislocations (Wiley, New York, 1982), 2nd ed.

[3] G.E. Beltz and L. B. Freund, Philos. Mag. A 69, 171 (1994); K. Ohsawa, H. Koizumi, O. K. Kirchner, and T. Suzuki, Philos. Mag. A 69, 183 (1994).

[4] I. L. F. Ray and D. J. H. Cockayne, Proc. R. Soc. London A 325, 543 (1971); A. Olsen and J. C. H. Spence, Philos. Mag. A 43, 945 (1980); J. L. Demenet et al., Philos. Mag. A 59, 501 (1989).

[5] T. A. Arias and J.D. Joannopoulos, Phys. Rev. Lett. 73, 680 (1994).

[6] K. Stokbro, N. Chetty, K. W. Jacobsen, and J. K. Nørskov, Phys. Rev. B 50, 10727 (1994).

[7] S. Goedecker and L. Colombo, Phys. Rev. Lett. 73, 122 (1994).

[8] E. Kaxiras and M. S. Duesbery, Phys. Rev. Lett. 70, 3752 (1993).

[9] B. Joós, Q. Ren, and M. S. Duesbery, Phys. Rev. B 50, 5890 (1994).

[10] J. R. K. Bigger et al., Phys. Rev. Lett. 69, 2224 (1992).

[11] L. B. Hansen, K. Stokbro, and B. I. Lundqvist [Mater. Sci. Eng. B (to be published)].

[12] M. S. Duesbery and G. Y. Richardson, Crit. Rev. Solid State Mater. Sci. 17, 1 (1991).

[13] A. George and J. Rabier, Rev. Phys. Appl. 22, 207 (1987).

[14] M. S. Duesbery, B. Joós, and D. J. Michel, Phys. Rev. B 43, 5143 (1991).

[15] P. B. Hirch, J. Phys. (France) IV 40, C6-27 (1979).

[16] R. Jones, J. Phys. (France) IV 40, C6-33 (1979).

[17] L. B. Hansen, K. Stokbro, B. I. Lundqvist, K. W. Jacobsen, and D. M. Deaven (to be published).

[18] K. W. Jacobsen, J. K. Nørskov, and M. J. Puska, Phys Rev. B 35, 7423 (1987); K. W. Jacobsen, Comments Condens. Matter Phys. 14, 129 (1988).

[19] N. Chetty, K. W. Jacobsen, and J. K. Nørskov, J. Phys. Condens. Matter 3, 5437 (1991).

[20] O. K. Andersen and O. Jepsen, Electronic Bandstructure and its Applications, edited by M. Yussouff (Springer Lecture Notes, New York, 1987).

[21] K. Stokbro, Ph.D. thesis, Technical University of Denmark, 1994.

[22] S. Goedecker and A. Teter, Cornell Theory Center report, 1994.

[23] J. W. Christian and V. Vitek, Rep. Prog. Phys. 33, 307 (1970); V. Vitek and M. Yamaguchi, J. Phys. F 3, 537 (1973).

[24] J. Huang, M. Meyer, and V. Pontikis, Phys. Rev. B 42, 5495 (1990).

[25] V.L. Indendom, in Elastic Strain Fields and Dislocation Mobility, edited by V. L. Indenbom and J. Lothe, Modern Problems in Condensed Matter Sciences Vol. 31 (NorthHolland, Amsterdam, 1992) 